\title{
Homogeneous ice nucleation at moderate supercooling from molecular simulation
}

\author{
E. Sanz, C. Vega, J. R. Espinosa, R. Caballero-Bernal, J. L. F. Abascal and C. Valeriani \\ Departamento de Química Física, Facultad de Ciencias Químicas, \\ Universidad Complutense de Madrid, 28040 Madrid, Spain
}

(Dated: December 4, 2013)

\begin{abstract}
Among all the freezing transitions, that of water into ice is probably the most relevant to biology, physics, geology or atmospheric science. In this work we investigate homogeneous ice nucleation by means of computer simulations. We evaluate the size of the critical cluster and the nucleation rate for temperatures ranging between $15 \mathrm{~K}$ and $35 \mathrm{~K}$ below melting. We use the TIP4P/2005 and the TIP4P/Ice water models. Both give similar results when compared at the same temperature difference with the model's melting temperature. The size of the critical cluster varies from $\sim 8000$ molecules (radius $=4 \mathrm{~nm}$ ) at $15 \mathrm{~K}$ below melting to $\sim 600$ molecules (radius $=1.7 \mathrm{~nm}$ ) at $35 \mathrm{~K}$ below melting. We use Classical Nucleation Theory (CNT) to estimate the ice-water interfacial free energy and the nucleation free energy barrier. We obtain an interfacial free energy of $29(3) \mathrm{mN} / \mathrm{m}$ from an extrapolation of our results to the melting temperature. This value is in good agreement both with experimental measurements and with previous estimates from computer simulations of TIP4P-like models. Moreover, we obtain estimates of the nucleation rate from simulations of the critical cluster at the barrier top. The values we get for both models agree within statistical error with experimental measurements. At temperatures higher than $20 \mathrm{~K}$ below melting we get nucleation rates slower than the appearance of a critical cluster in all the water of the hydrosphere in the age of the universe. Therefore, our simulations predict that water freezing above this temperature must necessarily be heterogeneous.
\end{abstract}

\section{INTRODUCTION}

When a liquid is cooled below its freezing point it is supposed to freeze. Usually, impurities or the solid boundaries of the liquid provide preferential sites for the formation of the solid phase. However, even in the absence of impurities, small nuclei of the new phase may be formed within the bulk metastable liquid. This mechanism of formation of the solid phase is called homogeneous nucleation. 1, 2] Homogeneous nucleation is an activated process since the formation of a critical nucleus requires the surmounting of a free energy barrier. After that, the crystalline nucleus can grow (nucleationand-growth mechanism). In general, at moderate supercooling, the limiting step is the formation of the critical cluster rather than the crystal growth. The most relevant quantity to characterize nucleation is the nucleation rate, i.e. the number of nucleating clusters per unit time and volume.

Water freezing is arguably the most important liquidto-solid transition. For example, ice formation in atmospheric clouds is a key factor to the global radiation budget and to climate change. [3 [5] Water freezing is also a big issue, for instance, in the cryopreservation of cells and tissues. [6] Moreover, ice formation is relevant to microbiology [7], food industry [8, 9], materials science [10], geology 11] or physics. 1, 12 17]

Despite its great importance, our understanding of water freezing is far from complete. Not even homogeneous nucleation, the simplest conceivable mechanism by which ice can be formed, is fully understood. One of the reasons for this is the need to perform experiments with small droplets $(10-100 \mu \mathrm{m})$ to avoid heterogeneous nucleation. 18 21] This, and the time that the droplets can be stabilized, sets the order of magnitude that can be probed for the nucleation rate, $J$. Thus, experimental measurements for $\log _{10}\left(\mathrm{~J} /\left(\mathrm{m}^{-3} \mathrm{~s}^{-1}\right)\right)$ typically range between 4 and and 14 . This corresponds to a temperature window spanning from $239 \mathrm{~K}$ to $233 \mathrm{~K}$, the latter often referred to as "homogeneous nucleation temperature". 22 Our knowledge of the nucleation rate outside this temperature window is limited to extrapolations based on CNT. Such extrapolations must be taken with care since the uncertainties in the nucleation rate and the narrow range of temperatures for which $\mathrm{J}$ can be measured lead to important differences in the estimated value of the interfacial free energy and/or the kinetic prefactor. [20] Moreover, it has not been possible so far to observe a critical ice nucleus in experiments because critical nuclei are relatively small and short-lived. Therefore, we only have estimates of the critical cluster size based on experimental measurements of $J$. 12, 18, 23 26] The purpose of this paper is to fill these gaps by obtaining the first estimate of the size of the critical cluster and of the nucleation rate at high temperatures which is not entirely based on theoretical extrapolations from measurements at low temperatures. We will make use of computer simulations to achieve these goals.

Computer simulations are a valuable tool to investigate nucleation 27, 28] since they provide a microscopic description of the process. It is therefore somehow surprising that the number of simulation studies dealing with ice nucleation is rather small. 29] On the one hand, it has been shown that ice nucleation can occur spontaneously (without the aid of special simulation techniques) when an electric field is applied 30], when crystallization is assisted by a substrate [31, 32] or by an interface 33], when coarse-grained models with accelerated dynamics are simulated at high supercoolings, 15, 34, 35] or when 
small systems are simulated 36 38]. On the other hand, if nucleation does not happen spontaneously, rare event techniques must be used. The number of such works is limited and the agreement between different groups is not entirely satisfactory. Radhakrishnan and Trout 39, 40], Quigley and Rodger 41] and Brukhno et al. 42] determined the free energy barrier for the formation of ice critical clusters with the TIP4P water model at $180 \mathrm{~K}$ (50 degrees below the model's melting temperature), but mutually consistent results were not found. Reinhardt and Doye [43] and Li et al. [16] evaluated the nucleation rate of the $\mathrm{mW}$ model at $55 \mathrm{~K}$ below freezing finding a discrepancy of six orders of magnitude. Very recently, Reinhardt et al. investigated ice nucleation at moderate supercoolings, 44 to estimate the free energy of formation of small pre-critical clusters. It is almost certain that more ice nucleation studies are on the way and, hopefully, the discrepancies will become smaller.

None of the studies mentioned in the previous paragraph deal with large systems at moderate supercoolings like the present investigation does. By supercooling, $\Delta T$, we mean the difference between the melting temperature and the temperature of interest. Note that the melting temperature of a model does not necessarily coincide with the experimental melting temperature or with the melting temperature of other models. In this work we determine, by means of computer simulations, the size of critical ice clusters and the nucleation rate for $\Delta T$ ranging from 15 to $35 \mathrm{~K}$. In this way we provide, for the first time, nucleation rates for $\Delta T$ lower than $35 \mathrm{~K}$, where experimental measurements are not currently feasible (CNT based estimates of $J$ can in principle be made for any supercooling but, to the best of our knowledge, there are no such estimates available for $\Delta T<30 K)$. [2, 12, 20] Our simulations predict that for $\Delta T<20 \mathrm{~K}$ it is impossible that homogeneous ice nucleation takes place. Therefore, ice must necessarily nucleate heterogeneously for supercoolings lower than $20 \mathrm{~K}$. Moreover, we can directly compare our results for the largest studied supercoolings to the experimental measurements. We find, within uncertainty, a good agreement with experimental nucleation rates. We predict that the radius of the critical cluster goes from $\sim 40 \AA$ (8000 molecules) at $\Delta T$ ca. $15 \mathrm{~K}$ to $\sim 17 \AA(600$ molecules $)$ at $\Delta T$ ca. $35 \mathrm{~K}$. We also estimate the surface free energy via CNT. We obtain, in agreement with predictions based on experimental measurements, [12, 45, 46] that the surface free energy decreases with temperature. An extrapolation of the interfacial free energy to the melting temperature gives a value of $\sim 29 \mathrm{mN} / \mathrm{m}$, in reasonable agreement with experimental results [47], and with calculations by simulation. [48]

We use two simple, yet realistic, water models; namely TIP4P/2005 49] and TIP4P/Ice 50]. The melting temperature [49, 50] and the ability of these models to predict properties of real water has already been well established. 51] The results obtained for both water models are quite similar provided that they are compared at the same $\Delta T$.

\section{METHODOLOGY}

To evaluate the size of critical ice clusters we follow a similar approach to that proposed by Bai and $\mathrm{Li}[52]$ to calculate the solid-liquid interfacial energy for a Lennard-Jones system. They employ spherical crystal nuclei embedded in the supercooled liquid and determine the temperature at which the solid neither grows nor melts. The key issue of this methodology is that determining the melting temperature of a solid cluster embedded in its corresponding supercooled liquid water is equivalent to the determination of the critical size of the cluster for a certain given temperature. Thus, in a sense, this methodology can be regarded as the extension to nucleation phenomena of the well known direct coexistence technique. 53] A similar method was applied to water by Pereyra et al. 54 They inserted an infinitely long (through periodical boundary conditions) ice cylinder in water and determined the melting temperature of the cylinder. Recently, the approach of Bai and $\mathrm{Li}$ has been used to investigate the nucleation of clathrate hydrates. 55, 56.

Here we shall implement this methodology to study a three-dimensional spherical ice cluster embedded in supercooled water. This follows closely the experimental situation where the incipient ice embryo is fully immersed into liquid water. Such brute force approach requires very large systems (containing up to $2 \times 10^{5}$ water molecules). However, molecular dynamics simulations can be efficiently parallelised so that it is nowadays possible to deal with such system size. The methodology can then be implemented in a rather straightforward way, and is particularly useful at moderate supercooling, where other techniques (such as umbrella sampling [57, 58], Forward Flux Sampling [59] or Transition Path Sampling [60]) may become numerically too expensive.

Once we calculate the critical cluster size we make use of CNT 61 63] in its version for spherical clusters to estimate the surface free energy, $\gamma$ :

$$
\gamma=\left(\frac{3 N_{c} \rho_{s}^{2}|\Delta \mu|^{3}}{32 \pi}\right)^{1 / 3}
$$

where $\rho_{s}$ is the number density of the solid and $\Delta \mu$ is the chemical potential difference between the metastable liquid and the solid at the temperature under consideration. This expression allows us to obtain a value for $\gamma$ associated to each cluster. CNT can also be used to estimate the height of the nucleation free-energy barrier, $\Delta G_{c}:$

$$
\Delta G_{c}=\frac{16 \pi \gamma^{3}}{3 \rho_{s}^{2}|\Delta \mu|^{2}} .
$$

Once $\Delta G_{c}$ is known, we can use the following CNT-based expression to evaluate the nucleation rate 64$]$ :

$$
J=Z f^{+} \rho_{f} \exp \left(-\Delta G_{c} /\left(k_{B} T\right)\right)
$$


where $Z$ is the Zeldovich factor, $Z=$ $\sqrt{\left(\left|\Delta G^{\prime \prime}\right|_{N_{c}} /\left(2 \pi k_{B} T\right)\right)}$, and $f^{+}$is the attachment rate of particles to the critical cluster. The CNT form of the Zeldovich factor is

$$
Z=\sqrt{|\Delta \mu| /\left(6 \pi k_{B} T N_{c}\right)}
$$

which can be obtained from our calculations of $N_{c}$. We follow Ref. 64 to calculate $f^{+}$as a diffusion coefficient of the cluster at the top of the barrier:

$$
f^{+}=\frac{<\left(N(t)-N_{c}\right)^{2}>}{2 t} .
$$

Therefore, in order to obtain nucleation rates we combine CNT predictions with simulations of the critical clusters.

By using the methodology here described, the nucleation rate of clathrate hydrates has been recently calculated. [56] The validity of this approach relies on the ability of CNT to make good estimates of the free energy barrier from measured values of the critical cluster size. CNT is expected to work well for big critical clusters. We are confident that the cluster sizes we deal with in this work are big enough for CNT to produce meaningful predictions. We discuss why in Sec. VA

\section{TECHNICAL DETAILS}

\section{A. Simulation details}

We carry out $N p T$ GROMACS 65] molecular dynamics simulations (MD) of a system that consists of one spherical ice-Ih cluster surrounded by supercooled water molecules. We use two different rigid non-polarizable models of water: TIP4P/2005 49 and TIP4P/Ice. 50] TIP4P/2005 is a model that provides a quantitative account of many water properties [51, 66] including not only the well known thermodynamic anomalies but also the dynamical ones. 67, 68 TIP4P/Ice was designed to reproduce the melting temperature, the densities and the coexistence curves of several ice phases. One of the main differences between the two models is their ice Ih melting temperature at 1 bar: $T_{m}=252 \mathrm{~K}$ for TIP4P $/ 2005$ and $T_{m}=272 \mathrm{~K}$ for TIP4P/Ice. We evaluate long range electrostatic interactions using the smooth Particle Mesh Ewald method [69] and truncate both the LJ and real part of the Coulombic interactions at $9 \AA$. We preserve the rigid geometry of the water model by using constraints. All simulations are run at the constant pressure of $p=1$ bar, using an isotropic ParrinelloRahman barostat 70 and at constant temperature, using the velocity-rescaling thermostat. 71] We set the MD time-step to 3 fs.

\section{B. Order parameter}

To determine the time evolution of the cluster size, we use the rotationally invariant order parameters proposed by Lechner and Dellago, $\bar{q}_{i} \cdot 72$. In Fig. 1 we show the $\bar{q}_{4}, \bar{q}_{6}$ values for 5000 molecules of either liquid water, ice Ih or ice Ic at 1 bar and $237 \mathrm{~K}$ for TIP4P/2005. The cut-off distance to identify neighbors for the calculation of $\bar{q}_{i}$ is $3.5 \AA$ between the oxygen atoms. This approximately corresponds to the position of the first minimum of the oxygen-oxygen pair correlation function in the liquid phase.

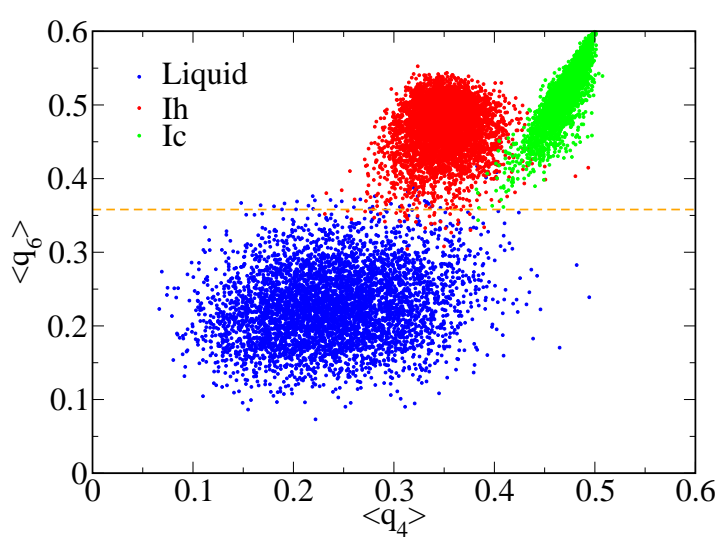

FIG. 1: Values of $\bar{q}_{6}$ and $\bar{q}_{4}$ [2] for 5000 molecules of the liquid phase (blue), of ice-Ih (red), and of ice-Ic (green) at $237 \mathrm{~K}$ for the TIP4P/2005 model.

From Fig. 1 it is clear that $\bar{q}_{6}$ alone is enough to discriminate between solid-like and fluid-like molecules, as already suggested in Ref. 73. As a threshold to separate the liquid from the solid clouds in Fig. 1 we choose $\bar{q}_{6, t}=0.358$, represented as a horizontal dashed line in the figure. This threshold separates the liquid from both ice Ih and Ic. Therefore, even though we prepare the clusters with ice-Ih structure, ice-Ic molecules would be detected as solid-like should they appear as the clusters grow. Unlike Refs. 74 and 75 we do not consider as solidlike particles on the surface which are neighbor to solidlike particles. Once molecules are labelled either as solid or liquid-like, the solid cluster is found by means of a clustering algorithm that uses a cut-off of $3.5 \AA$ to find neighbors of the same cluster.

\section{Initial configuration}

We prepare the initial configuration by inserting a spherical ice-Ih cluster (see Fig. 2 for a cluster of 4648 molecules) into a configuration of supercooled water with $\sim 20$ times as many molecules as the cluster. To obtain the cluster, we simply cut a spherical portion of a large equilibrated ice Ih crystal. Next, we insert the ice cluster in the supercooled liquid removing the liquid molecules that overlap with the cluster. Finally, we equilibrate the system for about 0.2 ns at $200 \mathrm{~K}$. This time is long enough to equilibrate the cluster-liquid interface (see Supporting Information). We then perform simulations for three different system/cluster sizes labeled as 


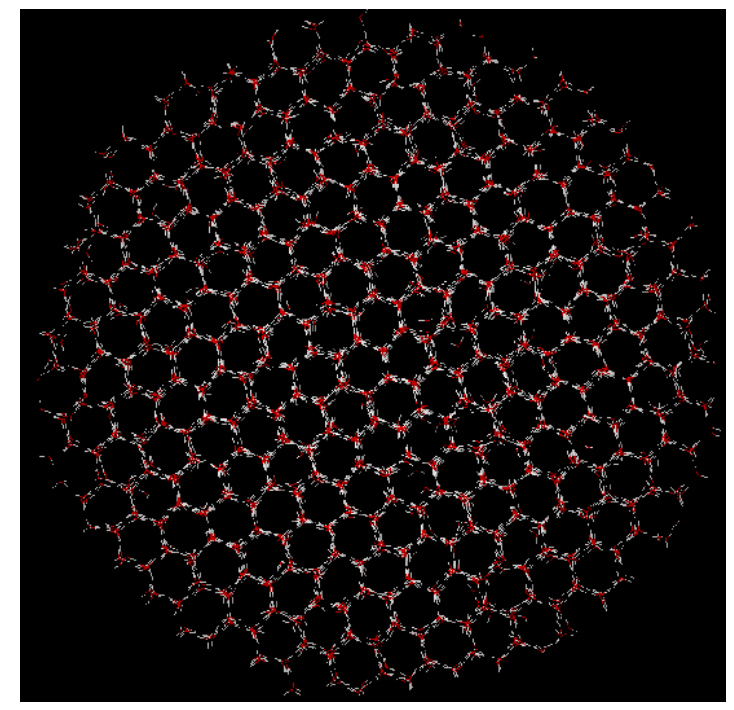

FIG. 2: Snapshot of a spherical ice-Ih cluster of 4648 molecules.

H (Huge), L (Large) and B (Big) (see Table \). As far as we are aware, the studied system size are beyond any previous numerical study of ice nucleation. Calculations were performed in the Spanish super-computer Tirant. For system $\mathrm{H}$ we use 150 nodes yielding $0.72 \mathrm{~ns} /$ day; for system L, 50 nodes at $1.5 \mathrm{~ns} /$ day and, for system B, 32 nodes at $4.7 \mathrm{~ns} /$ day.

Our order parameter allows us to correctly identify as solid-like the great majority of the molecules belonging to the cluster shown in Fig. 2 (4498 out of 4648). Fig. 3)(a) shows that indeed most molecules of the inserted ice cluster are detected as solid-like (red) as opposed to liquidlike (blue). Notice that most blue particles in Fig. [3(a) are located at the interface. This is not surprising giving that our order parameter was tuned to distinguish between liquid-like and solid-like particles in the bulk. Fig. 31(a) corresponds to the cluster just inserted in the liquid. After $0.2 \mathrm{~ns}$ of equilibration our order parameter detects that the number of molecules in the cluster drops down to 3170 . To explain the origin of this drop we show in Fig. 3.(b) a snapshot of the 4648 inserted molecules after the 0.2 ns equilibration period. Clearly, the drop comes from the fact that the outermost layer of molecules of the inserted cluster becomes liquid-like during equilibration. By removing the liquid-like molecules from Fig. 3(b) one can easily identify again the hexagonal channels typical of ice (Fig. 3(c)). Therefore, the drop from 4648 to 3170 molecules in the ice cluster is due to the equilibration of the ice-water interface. The size of the equilibrated clusters, $N_{c}$, is given in Table \

Once the interface is equilibrated for $0.2 \mathrm{~ns}$, the number of molecules in the cluster grows or shrinks (depending on the temperature) at a much slower rate (typically requiring several nanoseconds as it is shown in Fig. 4). The initial time in our simulations corresponds to the configuration equilibrated after 0.2 ns. We run MD sim-
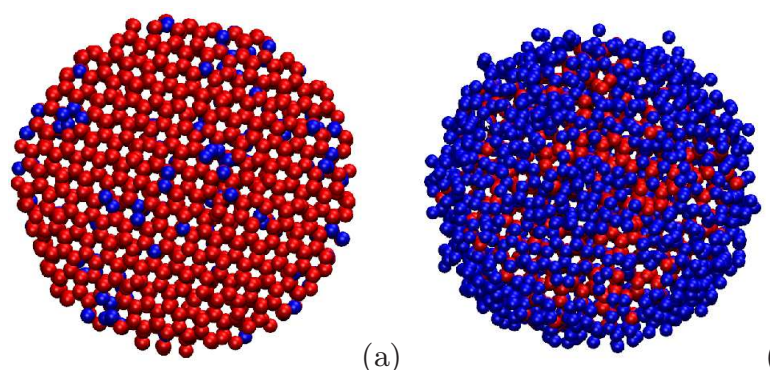

(a)

(b)

FIG. 3: Snapshot of the 4648 molecules inserted as an ice cluster just after insertion (a), and after 0.2 ns equilibration (b). Molecules are colored in red if detected as solid-like and in blue if detected as liquid-like. In (c) only solid-like molecules of snapshot (b) are shown.

TABLE I: Total number of molecules in the system, $N_{t}$ (ice cluster + surrounding liquid water molecules) and number of molecules of the inserted spherical ice cluster, $N_{i}$ for the three configurations prepared. $N_{c}$ is the number of molecules in the ice cluster after equilibration of the interface. The radius of the equilibrated clusters $r_{c}$ in $\AA$ is also presented.

\begin{tabular}{ccccccc}
\hline System & $N_{t}$ & $N_{i}$ & $N_{c}^{2005}$ & $N_{c}^{I c e}$ & $r_{c}^{2005}$ & $r_{c}^{\text {Ice }}$ \\
\hline $\mathrm{B}$ & 22712 & 1089 & 600 & 600 & 16.7 & 16.8 \\
$\mathrm{~L}$ & 76781 & 4648 & 3170 & 3167 & 29.1 & 29.2 \\
$\mathrm{H}$ & 182585 & 9998 & 7931 & 7926 & 39.5 & 39.7 \\
\hline
\end{tabular}

ulations of the system with the equilibrated interface at several temperatures below the bulk melting temperature of the model. The objective is to find a temperature range within which the cluster can be considered to be critical. The temperature range is comprised between the lowest temperature at which the solid cluster melts and the highest at which it grows. We monitor the number of molecules in the cluster and the global potential energy to find whether the cluster melts or grows.

\section{RESULTS}

\section{A. Size of the critical clusters}

In Fig. 4 we represent the number of molecules in the ice cluster versus time for system H, TIP4P/2005. Depending on the temperature the cluster either grows (230 K and $235 \mathrm{~K}$ ) or shrinks $(240 \mathrm{~K})$. The highest temperature at which the cluster grows is $235 \mathrm{~K}$ and the lowest temperature at which it melts is $240 \mathrm{~K}$. Hence, 


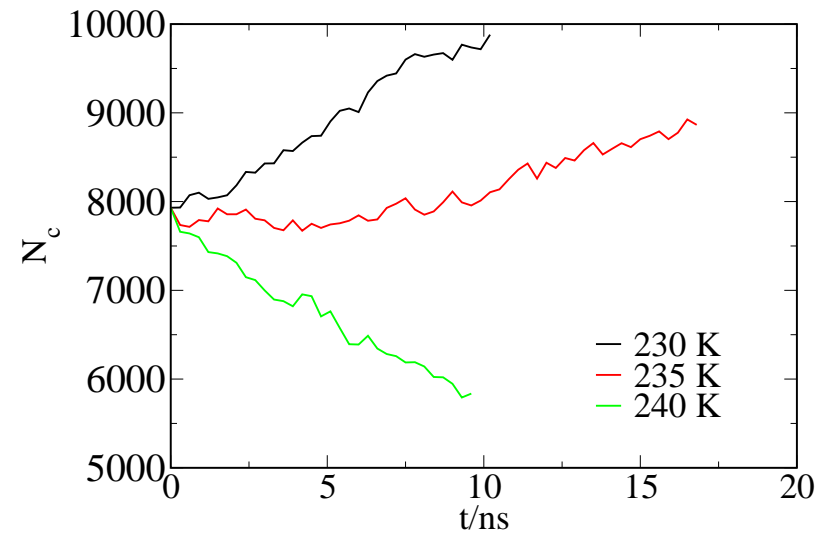

FIG. 4: Number of molecules in the ice cluster versus time for system $\mathrm{H}$ and the TIP $4 \mathrm{P} / 2005$ potential. Results are shown for different temperatures as indicated in the legend.

a cluster of $\sim 7900$ molecules (as detected by our order parameter) is critical at $237.5 \pm 2.5 \mathrm{~K}$. An analogous result can be obtained by monitoring the potential energy of the system as a function of time. (see Supporting Information). A decrease in the energy corresponds to the cluster's growth whereas an increase in the energy corresponds to its melting. By doing this analysis for both models (TIP4P/2005 and TIP4P/Ice ) and for the three cluster sizes (H, L, and B ), we obtain the results summarized in Table 【]

TABLE II: We report the temperature ( $T$ in $\mathrm{K}$ ) for which the prepared ice clusters are found to be critical, the supercooling ( $\Delta T$ in $\mathrm{K}$ ) for the corresponding water model, the chemical potential difference between the fluid and the solid $(\Delta \mu$ in $\mathrm{kcal} / \mathrm{mol})$, the liquid-solid surface free energy $(\gamma$ in $\mathrm{mN} / \mathrm{m})$ estimated from Eq. 1 and the nucleation free energy barrier height $\left(\Delta G_{c}\right.$ in $\left.k_{B} T\right)$ estimated from Eq. 2

\begin{tabular}{cccccccc}
\hline Model & System & $N_{c}$ & $T$ & $\Delta T$ & $\Delta \mu$ & $\gamma$ & $\Delta G_{c}$ \\
\hline TIP4P/2005 & $\mathrm{B}$ & 600 & 222.5 & 29.5 & 0.114 & 20.4 & 77 \\
TIP4P/2005 & $\mathrm{L}$ & 3170 & 232.5 & 19.5 & 0.080 & 24.9 & 275 \\
TIP4P/2005 & $\mathrm{H}$ & 7931 & 237.5 & 14.5 & 0.061 & 25.9 & 515 \\
\hline TIP4P/Ice & $\mathrm{B}$ & 600 & 237.5 & 34.5 & 0.133 & 23.6 & 85 \\
TIP4P/Ice & $\mathrm{L}$ & 3167 & 252.5 & 19.5 & 0.083 & 25.4 & 261 \\
TIP4P/Ice & $\mathrm{H}$ & 7926 & 257.5 & 14.5 & 0.063 & 26.3 & 487 \\
\hline
\end{tabular}

For the temperatures explored in this work (from about $15 \mathrm{~K}$ to $35 \mathrm{~K}$ below the melting temperature of both TIP4P/2005 and TIP4P/Ice) the size of the ice critical cluster ranges from nearly 8000 (radius of $4 \mathrm{~nm}$ ) to about 600 molecules (radius of $1.7 \mathrm{~nm}$ ). This compares reasonably well with a critical cluster radius of $\sim 1.3 \mathrm{~nm}$ obtained by applying CNT to experimental measurements at a supercooling of about $40 \mathrm{~K} .[25$, 26] Our results are also consistent with CNT based estimates of the critical size at lower supersaturations. 2, 76] For instance, in Fig. 15.7 of Ref. [2], a critical cluster size ranging from 1000 to 300 molecules is predicted for $25 \mathrm{~K}<\Delta T<$
$30 \mathrm{~K}$. An interesting remark is that the temperatures of the TIP4P/Ice are basically shifted $20 \mathrm{~K}$ above the the corresponding ones for TIP $4 \mathrm{P} / 2005$ with the same nucleus size. This is precisely the difference between the melting temperatures of both models and, thus, the supercoolings are very similar for a given ice cluster size in both models. This is more clearly shown in Fig. 5 where the size of the critical cluster is plotted as a function of the the difference between the melting temperature of the model and the temperature of interest $\Delta T=T_{m}-T$. We observe that, within our error bar, the critical cluster size of both models scales in the same way with respect to their melting temperatures. This is not so surprising since TIP4P/2005 and TIP4P/Ice present a similar charge distribution and mainly differ in the choice of the potential parameters.

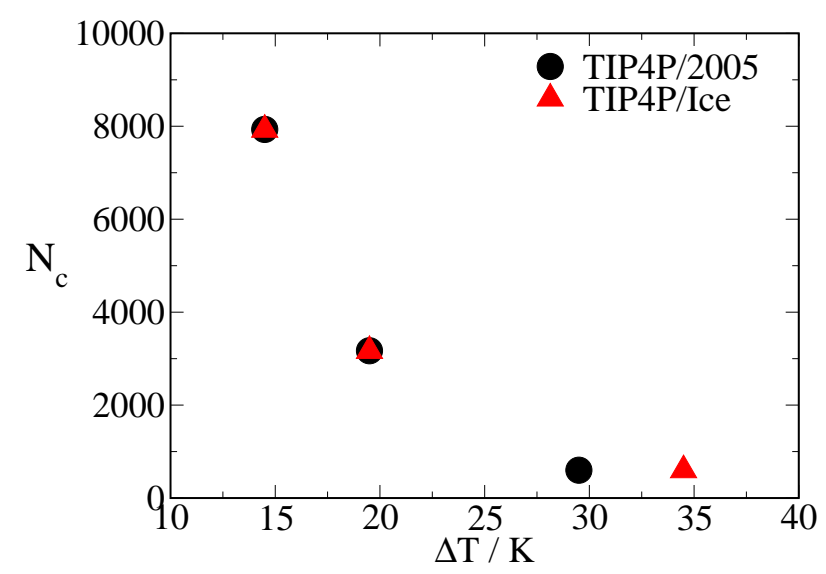

FIG. 5: Critical cluster size versus $\Delta T$ for the studied water models. Notice that the points corresponding to both models at low supercooling are essentially on top of each other.

In previous works [51, 66, 77] we observed that, for a number of properties, the values of TIP4P/2005 lie in the middle of the values obtained for TIP4P and TIP4P/Ice. Therefore, it is expected that TIP4P gives similar results to TIP4P/2005 and TIP4P/Ice regarding the dependence of $N_{c}$ with $\Delta T$. Matsumoto et al. [36] studied ice nucleation at $230 \mathrm{~K}$ and a density of $0.96 \mathrm{~g} / \mathrm{cm}^{3}$ using the TIP4P model. This thermodynamic state point corresponds to a pressure of about -1000 bar and $\Delta T 5 \mathrm{~K}$. 78. By extrapolating the data of Fig. 5 to $\Delta T=5 K$ one gets a critical cluster of the order of hundreds of thousand molecules. Therefore, it is likely that the results obtained by Matsumoto et al. 36], although pioneering and useful to learn about the ice nucleation pathway, may suffer from system size effects and may not be valid to estimate either the size of the critical cluster or the nucleation rate.

In an important paper, Koop et al. [79] showed that the homogeneous nucleation rate (and therefore the temperature of homogeneous nucleation) of pure water and of water solutions can be described quite well by a function that depends only on the water activity. This conclusion has been confirmed in more recent experiments. 14 
Although the nucleation rate for an aqueous solution is the same as for pure water, the freezing points are different. One is then tempted to suggest that the size of the critical cluster at the homogeneous nucleation temperature could be the same for pure water and for aqueous solutions. Moreover, the fact that thermodynamics is sufficient to predict the rate seems to indicate that the water mobility is also determined by the free energy of water. A microscopic study of the relationship between crystallization rates, structure and thermodynamics of water which may explain the empirical findings of Koop and coworkers has recently been presented in Ref. 15 .

\section{B. Interfacial free energy and free energy barrier}

Once the size of the critical cluster is known, one can use Eq. 1 to estimate the solid-liquid interfacial free energy. Since ice density changes little with temperature [80], the density at coexistence is used in our calculations $\left(\rho_{\text {m,TIP4P/Ice }}=0.906 \mathrm{~g} / \mathrm{cm}^{3}\right.$ and $\left.\rho_{m, T I P 4 P / 2005}=0.921 \mathrm{~g} / \mathrm{cm}^{3}\right)$. For most substances it is possible to approximate $\Delta \mu$ by $\Delta h_{m}\left(T_{m}-T\right) / T_{m}$, where $\Delta h_{m}$ is the melting enthalpy and $T_{m}$ is the melting temperature. For water, however, this may not be a good approximation because $\Delta h$ significantly changes with temperature as a manifestation of the anomalous sharp increase of the heat capacity of water as temperature decreases. 24, 81] Hence, one needs to do a proper evaluation of the chemical potential difference between both phases to get the surface free energy from Eq. 1. We have calculated $\Delta \mu$ at every temperature by means of standard thermodynamic integration [82] from the coexistence temperature, at which $\Delta \mu=0$. In Table 1 we report the values we obtain for $\Delta \mu$ and $\gamma$.

First of all we note that $\gamma$ decreases with temperature for both models. This is in qualitative agreement with experimental estimates of the behavior of $\gamma$ with $T .[12,45,46]$ A more quantitative comparison is not possible in view of the large discrepancies between different estimates (see Fig. 10 in Ref. 12). Motivated by the fact that the interfacial free energy can only be measured at coexistence, we extrapolate our results to the melting temperature. To do that, we take the two largest clusters and evaluate the slope of $\gamma(T)$. We get a value for the slope of $\sim 0.18 \mathrm{mN} /(\mathrm{m} \mathrm{K})$ for both models, in very good agreement with a recent calculation for the TIP4P/2005 model. [44] With a linear extrapolation we get a value for $\gamma$ at $T_{m}$ of $\sim 28.7 \mathrm{mN} / \mathrm{m}$ for both models, which can be compared to experimental measurements. In contrast with the vapor-liquid surface tension, the value of $\gamma$ for the solid-fluid interface is not well established. Experimental values range from 25 to $35 \mathrm{mN} / \mathrm{m}$. 83] Our calculated data for $\gamma$ at coexistence lies in the middle of that range, so our models predict a surface free energy which is consistent with current experimental data. We now compare our estimated $\gamma$ to direct calculations from simulations using a planar interface. The value of $\gamma$ de- pends on the plane in contact with the liquid. Since the cluster used here is spherical we shall compare with the average of the values obtained for the basal and prismatic planes. Davidchak et al. computed $\gamma$ for a planar fluid-solid interface using two models similar to those used in this work: TIP4P and TIP4P-Ew. For TIP4P, in an initial publication the authors reported a value of $\gamma=23.9 \mathrm{mN} / \mathrm{m}[84$ that was later on modified (after improving their methodology) to $\gamma=26.5 \mathrm{mN} / \mathrm{m}$. 48] For the TIP4P-Ew 85] Davidchak et al. reported (using the improved methodology) a value of $27.6 \mathrm{mN} / \mathrm{m}$. 48] TIP4P-Ew is known to predict water properties in relatively close agreement to those of TIP $4 \mathrm{P} / 2005$. Therefore, our results are also consistent with the calculations reported in the literature for similar models. To conclude, our values of $\gamma$ seem to be reasonable estimates of the interfacial free-energy of the planar ice-water interface.

To estimate the height of the nucleation free-energy barrier we make use of Eq. 2. Our results are summarized in Table In view of the height of the nucleation barrier for the clusters of systems $\mathrm{L}$ and $\mathrm{H}$, around 250 and 500 $k_{B} T$ respectively, it seems virtually impossible to observe homogeneous nucleation of ice for supercoolings lower than $20 \mathrm{~K}$. The height of the nucleation barrier provides an estimate of the concentration of critical clusters in the metastable fluid as $\rho_{f} \exp \left(-\Delta G_{c} /\left(k_{B} T\right)\right)$, where $\rho_{f}$ is the number density of the fluid. For $\Delta G_{c}=250 k_{B} T$, one critical cluster would appear on average in a volume $\sim 10^{60}$ times larger than the volume of the whole hydrosphere. From the values of $\Delta G_{c}$ of Table \we may infer why spontaneous ice nucleation has never been observed in previous studies of supercooled water with the TIP4P/2005 model [86 88]. Our results show that the free energy barrier for nucleation even for temperatures as low as $35 \mathrm{~K}$ below melting is still of about $80 k_{B} T$. This is much larger than the typical barrier found in studies where spontaneous crystallization occurs in brute force simulations [89, 90] (about $18 k_{B} T$ ). It is worth mentioning that neither Shevchuk and Rao 91] nor Overduin and Patey 92] find any evidence of ice nucleation in TIP4P models after runs of several microseconds which is consistent with the results of this work. Our results may be of great interest to studies in which the competition between the crystallization time and the equilibration time of water is crucial [93 95].

\section{Nucleation rate}

Although the free energy barriers alone provide a strong indication that ice can not appear on our planet via homogeneous nucleation at moderate supercoolings $(\Delta T<20 \mathrm{~K})$, it is worth calculating the nucleation rate, $J$, to confirm such statement. The nucleation rate takes into account not only the concentration of the clusters but also the speed at which these are formed. Moreover, the supercoolings for the smallest clusters we inves- 
tigate are comparable to those where most experimental measurements of $J$ have been made $(\Delta T \sim 35 \mathrm{~K}) .12$ 14, 17, 96 .

To calculate the nucleation rate we use Eq. 3. First, we compute $f^{+}$from Eq. 5 by running 30 simulations of the cluster at the temperature at which it was determined to be critical. We monitor $\left(N(t)-N_{c}\right)^{2}$ and average it over all the runs. In Fig. 6] we plot $<\left(N(t)-N_{c}\right)^{2}>$ versus time for the system L, TIP $4 \mathrm{P} / 2005$. From the slope at long times we can infer $f^{+}$.64 We get $f^{+}=$ $70 \cdot 10^{9} s^{-1}$. The Zeldovich factor for this particular case is $1.77 \cdot 10^{-3}$, and the density of the liquid is $0.977 \mathrm{~g} / \mathrm{cm}^{3}$. With this, we have all the ingredients needed to calculate the nucleation rate via Eq. 3. The final result for this case is $\log _{10}\left(\mathrm{~J} /\left(\mathrm{m}^{-3} \mathrm{~s}^{-1}\right)\right)=-83$.

The same procedure is used to calculate the nucleation rate for the rest of the systems described in Table I. The results for the nucleation rate as a function of the supercooling are presented in Fig. 7 and compared to the experimental measurements of Pruppacher 12 and Taborek[13]. The horizontal dashed line corresponds to the nucleation rate required for the appearance of one critical cluster in the volume of Earth's hydrosphere in the age of the universe, which we call "impossible nucleation rate". The vertical line shows at which temperature the impossible nucleation rate line intercepts the upper limit of our error bars (grey and orange shadows for TIP4P/2005 and TIP4P/Ice respectively). In view of this figure we can confidently claim what the free energy barriers previously hinted: it is impossible that ice nucleates homogeneously in our planet for $\Delta T<20 \mathrm{~K}$. In other words, heterogeneous nucleation must take place in order for water to freeze for supercoolings lower than $20 \mathrm{~K}$. This is consistent with the fact that, when heterogeneous nucleation is suppressed, moderately supercooled water can remain metastable long enough for its thermodynamic properties to be measured. 21, 22, 9799] From our results it is also clear that ice formation should not be expected in brute force molecular dynamics simulations at moderate supercoolings (provided that the system is large enough not to be affected by finite size effects). 36] To observe ice formation in brute force simulations the nucleation rate should be higher than $\log _{10}\left(\mathrm{~J} /\left(\mathrm{m}^{-3} \mathrm{~s}^{-1}\right)\right)=32$ (this number is obtained assuming the formation of ice after running about $100 \mathrm{~ns}$ in a system of about $50 \mathrm{~nm}^{3}$, which are typical values in computer simulations of supercooled water). Notice also that the maximum in the isothermal compressibility at room pressure 86, 87 found at about $\Delta T=20 \mathrm{~K}$ for the TIP4P/2005 model can not be the ascribed to the transient formation of ice as the nucleation rate of ice at this temperature is negligible.

Another interesting aspect of Fig. 7 is the comparison with experiment. Both models give nucleation rates that reproduce the experimental measurements within the uncertainty of our method. This excellent result brings confidence in the ability of the selected models to predict relevant quantities for the nucleation of ice such as the nucleation rate, the critical cluster size, and the surface free energy.

We also include in Fig. 7 a green dashed line that corresponds to the CNT based estimates of $J$ shown in Fig. 13.6 of Ref. 2] The agreement between CNT, simulations and experiments is quite satisfactory. To the best of our knowledge, there are no CNT estimates of $J$ available for supersaturations lower than $30 \mathrm{~K}$ to compare our results with. [2, 12, 20]

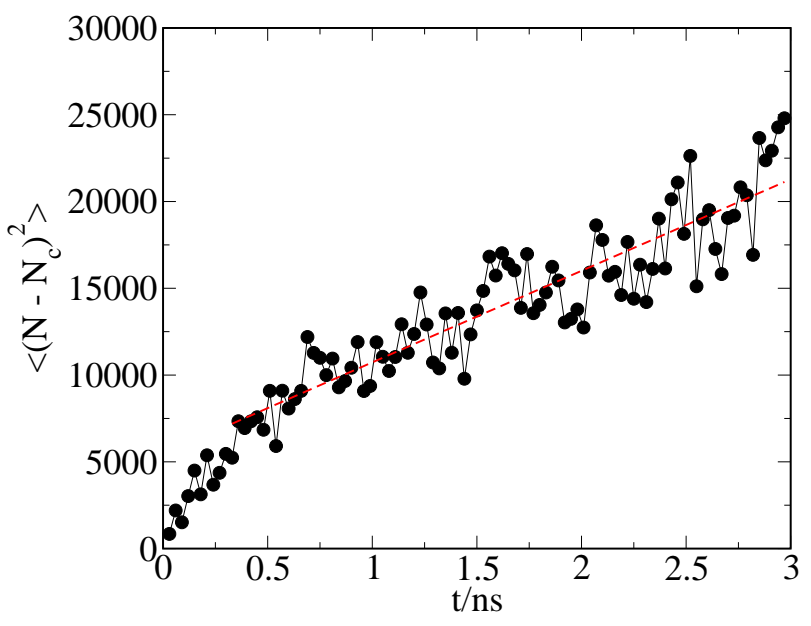

FIG. 6: $\left\langle\left(N(t)-N_{c}\right)^{2}>\right.$ versus time for configuration L, TIP $4 \mathrm{P} / 2005$. The attachment rate $f^{+}$is obtained as half the value of the slope. The curve above is obtained as an average over 30 trajectories. In approximately half of these trajectories the critical cluster ended up growing, whereas it eventually melted in the other half.

By using Forward Flux Sampling [59], Li et al. determined $J$ for the $\mathrm{mW}$ model of water for temperatures between 35 and $55 \mathrm{~K}$ below the model's melting temperature. [16] Since we are interested in ice nucleation at moderate supersaturation, our study deals with lower supercoolings $(14.5 \mathrm{~K}<\Delta T<34.5 \mathrm{~K})$. Nonetheless, our highest supercooling $(34.5 \mathrm{~K})$ is very close to the lowest one of $\mathrm{Li}$ et al. $(35 \mathrm{~K})$ so we can compare both results. The values of $\mathrm{Li}$ et al. for $J$ are 5-8 orders of magnitude below the experimental ones when compared at the same absolute temperature (the deviation increases when the comparison is made at the same degree of supercooling). The nucleation rates calculated in this work for TIP4P/2005 and TIP4P/ice are similar (although slightly larger) to those for the $\mathrm{mW}$ model. Initially this may appear surprising as the $\mathrm{mW}$ model is a coarse grained model of water with no hydrogens, which makes its dynamics faster than that of both real water and TIP4P-like models. 100 However the free energy barrier of $\mathrm{mW}$ may be larger, compensating this kinetic effect. In fact the interfacial free energy of $\mathrm{mW}$ has been found [16] to be $\gamma=31 \mathrm{mN} / \mathrm{m}$ (larger than the values found in this work for TIP4P/2005 and TIP4P/Ice). This high value of $\gamma$ may be partially compensated by a significant overestimate of the ice density of this model $\left(0.978 \mathrm{~g} / \mathrm{cm}^{3}\right.$ to be compared to the experimental result 


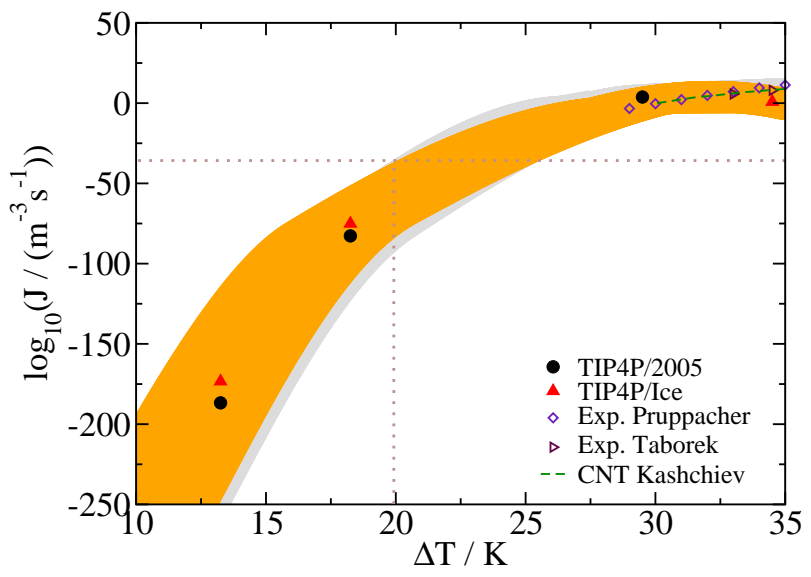

FIG. 7: Nucleation rate as a function of the supercooling. Symbols correspond to our simulation results and to experimental measurements as indicated in the legend. The green dashed line corresponds to CNT estimates of $J$.[2] The grey and orange shadows represent the estimated error bars for TIP4P/2005 and TIP4P/Ice respectively interpolated by splines. The horizontal dotted line indicates the rate given by the growth of one cluster in the age of the universe in all the water of the Earth's hydrosphere. The vertical dotted line indicates the supercooling below which homogeneous nucleation is impossible.

$\left.0.91 \mathrm{~g} / \mathrm{cm}^{3}\right)$. The net balance is that the values of $J$ of the $\mathrm{mW}$ model are similar, although somewhat lower, than those for TIP4P/2005 and TIP4P/ice.

As for the size of the critical cluster, we find that it is of about 600 water molecules for TIP4P/Ice at $237.5 \mathrm{~K}$ $(\Delta T=34.5 \mathrm{~K})$. Li et al. have reported a critical cluster size of about 850 molecules for the $\mathrm{mW}$ model at $240 \mathrm{~K}$ $(\Delta T=35 \mathrm{~K})$. Both results are compatible since Li et al. include in the ice cluster molecules which are neighbor to the solid cluster, and we do not. In summary, our results for TIP4P/2005 and TIP4P/Ice are consistent with Li's et al. for $\mathrm{mW}$.

\section{DISCUSSION}

\section{A. Validity and possible sources of error}

The methodology we have used is subject to two main error sources: the determination of the cluster size and the location of the temperature at which the clusters are found to be critical. Moreover, our approach relies on the validity of CNT. In the following paragraphs we discuss the extent to which our results may be affected by these issues.

In nucleation studies, the size of the largest solid cluster is usually considered a good reaction coordinate. To identify the cluster, we first need to distinguish between liquid-like and solid-like molecules. The chosen criterion should be able to identify the majority of molecules of the bulk solid as solid-like, and the majority of molecules of the bulk fluid as liquid-like. One could in principle find several criteria that successfully perform this task. However, when interfaces are present in the system (as in the case of a solid-liquid [89] or a solid-vapor [101, 102] interface) depending on the chosen criterion one might assign differently the interfacial molecules (see for instance References 43 and 16 for an illustration of this problem for the $\mathrm{mW}$ water model).

How does the choice of a criterion to distinguish liquid from solid-like molecules affect our results? Whether the cluster grows or shrinks for a given temperature does not depend on the particular choice of the order parameter (see Supporting Information). The same trend can be obtained by monitoring global thermodynamic properties of the system, such as the total potential energy (see Supporting Ingormation). Therefore, the fact that the cluster shown in Fig. 3 is critical at $232.5 \mathrm{~K}$ is independent on the particular choice of the criterion to distinguish liquid from solid-like molecules.

A different problem arises if one asks the question: how many ice molecules are present in Fig.3b? Different criteria provide different answers even though the configuration presented in Fig.3b is unique. Since the origin of this arbitrarity is due to the interfacial region, it is expected that the arbitrarity will become smaller as the ice cluster becomes larger. However, for the system sizes considered in this work the interface region still matters. To take this effect into account we have estimated the error bars in Fig. 7 considering an arbitrarity of $60 \%$ in the labeling of interfacial molecules. This would affect the value of $\gamma$ by $7 \%$, and the free-energy barriers height by up to $20 \%$. Although this estimated error seems large, it is worth pointing out that differences between the freeenergy barrier estimated by different groups may be, in the case of water, much larger than that. 39 42] In summary we conclude that the liquid/solid criterion chosen in this work provides reasonable estimates of $\gamma$, and when used within the CNT framework allows to interpret our simulations results in a rather straightforward way.

Another important error source in the calculation of $J$ is the location of the temperature at which a cluster is

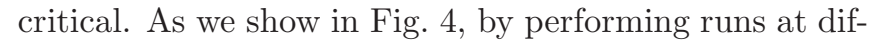
ferent temperatures we identify, within a certain range, the temperature that makes critical a given ice cluster. We assign the temperature in the middle of the range to the corresponding cluster, but the temperature that really makes the cluster critical could in principle be any other within the range. This uncertainty has a strong contribution to the error bars in Fig 7 , particularly at low supercoolings, where the variation of $J$ with $T$ is very steep. This error could, in principle, be easier to reduce than that coming from the arbitrarity in the determination of the number of particles in the cluster. One simply has to do more runs to narrow the temperature range. However, these simulations are very expensive given the large system sizes we are dealing with. It is interesting to point out that temperature control is also seen as a major error source in experiments. 17] 
Our results for $\gamma, \Delta G_{c}$, and $J$ rely on the validity of CNT. Classical Nucleation Theory is expected to break down for small clusters, when the view of nucleation as a competition between bulk and surface free energies starts to be questionable (in clusters of a few hundred particles most molecules are placed at the surface). However, for the large cluster sizes investigated in this work it seems reasonable to assume that CNT works well. The satisfactory comparison of our estimate of $\gamma$ with that obtained in simulations of a flat interface 48 is certainly encouraging in this respect. Moreover, we have applied the methodology described in this paper to calculate the nucleation rate of the $\mathrm{mW}$ water model and we get, within error, the same nucleation rate as in Ref.[16] This is a very stringent test to our approach, given that in Ref 16 ] a method that relies neither on CNT nor on the definition of the cluster size was used (Forward Flux Sampling). This comparison is made for a supercooling of $35 \mathrm{~K}$, the deepest investigated in this work. For lower supercoolings, where the critical cluster is larger, the methodology is expected to be even more robust. The advantage of the approach used here is that it allows to estimate (at a reasonable computational cost) critical cluster sizes and nucleation rates at low and moderate supercooling.

\section{B. Novelty}

In this paper we provide values for the homogeneous nucleation rate of ice at moderate supercoolings $(\Delta T<$ $33 \mathrm{~K}$ ). For the first time, this is done without extrapolating from measurements at high supercoolings. The experimental determination of $J$ is limited to a narrow temperature window at high supercoolings (between $233 \mathrm{~K}$ and $239 \mathrm{~K}$ ). In that window, $J$ can be directly measured without introducing any type of approximation. It only requires the knowledge of the droplet volume, the cooling rate and the fraction of freezing events. Differences in the value of $J$ between different experimental groups are relatively small (between one and two orders of magnitude). Therefore, the experimental value of $J$ is well established for the narrow range of temperatures in which the current experimental techniques can probe the nucleation rate. 12 14, 17, 96] To obtain values of $J$ outside that temperature window one can either extrapolate the data or make an estimate via CNT. An extrapolation from such a narrow temperature window would not be very reliable because $J$ changes sharply with T. In turn, an estimate of $J$ based on CNT relies in the knowledge of the interfacial free energy. Unfortunately, our current knowledge of $\gamma$ for the water-ice interface is far from satisfactory in at least three respects. Firstly, the calculated values of different groups using CNT differ significantly (see for instance Tables I and II in Ref 20). Secondly, the values obtained for $\gamma$ from CNT seem to be different from those determined for a planar ice-water interface at the melting point (see for instance Fig. 8 in Ref 103). Finally, there is even no consensus about the value of $\gamma$ for a planar interface at the melting point of water, a magnitude that in principle could be obtained from direct experiments without invoking CNT (values between 25 and $35 \mathrm{mN} / \mathrm{m}$ have been reported). A look to Fig. 10 of the classic paper of Pruppacher 12] is particularly useful. It shows the enormous uncertainty that exists at any temperature about the value of $\gamma$ for the ice-water interface. Since $\gamma$ enters in the estimation of $J$ as a power of three in an exponential term, the enormous scatter implies that, at this moment, there is no reliable estimate of the value of $J$ for moderately supercooled water arising from CNT. In other words, you can get many different estimates of $J$ from the different estimates of $\gamma$ shown in the paper by Pruppacher. In addition, to the best of our knowledge, no one has estimated $J$ using CNT for supersaturations lower than $30 \mathrm{~K}$. 2, 12, 20]

Regarding the critical nucleus size, it is not possible at the moment to measure it experimentally by direct observation. Therefore, the prediction of the critical cluster at moderate and experimentally accessible supercoolings is a novel result. Since the TIP4P/2005 has been quite successful in describing a number of properties of water (notably including the surface tension for the vapor-liquid equilibrium) we believe that the values reported here for $\gamma$ and $J$ from our analysis of the critical cluster are a reasonable estimate for the corresponding values for real water.

\section{Summary and outlook}

We have studied homogeneous ice nucleation by means of computer simulations using the TIP $4 \mathrm{P} / 2005$ and TIP4P/Ice water models. This is the first calculation of the size of the critical cluster and the nucleation rate at moderate supercoolings (14.5-35 K). Both models give similar results when compared at the same supercooling.

To determine the size of the critical cluster, we use a numerical approach in the spirit of direct coexistence methods. We prepare an initial configuration by inserting a large ice cluster (about 10000, 4600 and 1000 molecules) in an equilibrated sample of liquid water. Then, we let the interface equilibrate for $0.2 \mathrm{~ns}$ at $200 \mathrm{~K}$. Finally, we perform molecular dynamic runs at several temperatures to detect either the melting or the growth of the inserted cluster by monitoring its size. We find that the size of the critical cluster varies from $\sim 8000$ molecules (radius $=$ $4 \mathrm{~nm}$ ) at $15 \mathrm{~K}$ below melting to $\sim 600$ molecules (radius $=$ $1.7 \mathrm{~nm})$ at $35 \mathrm{~K}$ below melting.

We use CNT to estimate the interfacial free energy and the nucleation free energy barrier. Our predictions show that the interfacial free energy decreases as the supercooling increases, in agreement with experimental predictions. An extrapolation of the interfacial free energy to the melting temperature gives a value of $29(3) \mathrm{mN} / \mathrm{m}$, which is in reasonable agreement with experimental measurements and with estimates obtained from computer simulations for TIP4P-like models. We get free energy 
barriers higher than $250 \mathrm{kT}$ for supercoolings lower than $20 \mathrm{~K}$. This strongly suggests that homogeneous ice nucleation for supercoolings lower than $20 \mathrm{~K}$ is virtually impossible. We confirm this by calculating the nucleation rate. To do that we compute, by means of molecular dynamics simulations, the rate at which particles attach to the critical clusters. These calculations show that, indeed, for supercoolings lower than $20 \mathrm{~K}$ it is impossible that ice nucleates homogeneously. According to this prediction, ice nucleation must necessarily be heterogeneous for supercoolings lower than $20 \mathrm{~K}$. The nucleation rate we obtain at higher supercoolings (30-35 K) agrees, within the statistical uncertainty of our methodology, with experimental measurements.

It would be interesting to extend this work in several directions. Modifying the shape of the inserted cluster (inserting for instance a small crystal with planar faces) or even inserting a block of cubic ice Ic to analyse whether this cluster may be more stable as suggested by some studies 20, 104] are interesting issues that deserve further studies. Secondly, it would be of interest to consider other water models, to analyse the possible similarities/differences with respect to nucleation of different potential models varying significantly either in the charge distribution as TIP5P 105] or in the way the tetrahedral order is induced as in the $\mathrm{mW}$ model.100] Analyzing the behaviour at higher degrees of supercooling than those presented here is another interesting problem as well as the determination of the growth rate of ice. 106. We foresee that all these issues will be the centre of significant activity in the near future.

\section{SUPPORTING INFORMATION TO "HOMOGENEOUS ICE NUCLEATION AT MODERATE SUPERCOOLING FROM MOLECULAR SIMULATION"}

\section{A. Equilibration of the initial configuration}

The preparation protocol used for all cluster sizes is the following. After having inserted the ice cluster in the supercooled liquid, we remove the liquid molecules overlapping with the solid ones. Next, we equilibrate the system for about $0.2 \mathrm{~ns}$ at $200 \mathrm{~K}$. To make sure that the chosen $0.2 \mathrm{~ns}$ is a proper equilibration time, long enough to allow for annealing mismatches at the interface, we run a simulation starting from the initial configuration at time zero. In Fig. 8, we represent the cluster size for the Large system of the TIP4P/2005 model as a function of time. The figure shows that, even though the dynamics is very slow at such low temperatures, the chosen equilibration time is long enough. This result is independent on the chosen cluster size or water model potential.

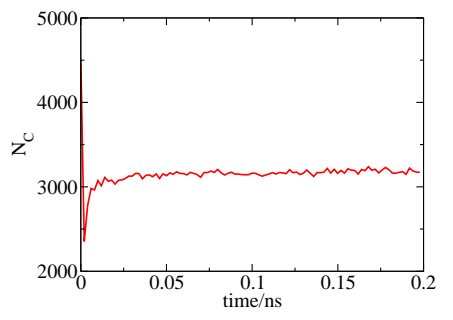

FIG. 8: Equilibration for the L system of the TIP4P/2005 model. After an initial drop of the cluster size due to equilibration of the interphase, the cluster size changes very slowly with time.

\section{B. Choice of the order parameter to distinguish between liquid/solid particles}

The use of an alternative order parameter to identify solid-like particles $\left(\bar{q}_{3}\right)$ does not affect the observed response of the cluster to temperature. This is shown in Fig. 9. where the number of particles in the cluster is monitored with two different order parameters for three different temperatures. Both order parameters allow to conclude that the inserted cluster is critical between 235 and $240 \mathrm{~K}$. Obviously, the number of particles that belong to the cluster does depend on the order parameter. The order parameter we use in this work should at least work well for the inner particles given that, according to Fig. 1 of the main text, it is able to discriminate between bulk liquid and bulk solid particles. The main ambiguity in the number of particles belonging to the cluster comes from those particles that lie in the interface. In view of Fig. 3 of the main text, it seems that our order parameter is doing reasonably well in identifying such particles either. Nonetheless, we have considered an error as large as $60 \%$ in the identification of the interfacial particles to estimate the error of the nucleation rate. In this way the unavoidable ambiguity in the determination of the cluster size is reflected in the error bar of $J$.

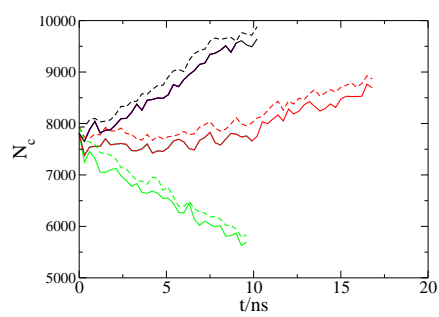

FIG. 9: Number of particles in the cluster versus time for system size $\mathrm{H}, \mathrm{TIP} 4 \mathrm{P} / 2005$ model. Black curves correspond to $230 \mathrm{~K}$, red ones to $235 \mathrm{~K}$ and green to $240 \mathrm{~K}$. Solid lines correspond to the analysis made with the order parameter described in the main text. Dashed lines correspond to the use of an alternative order parameter. With such order parameter particles are considered as neighbors if their oxygen atoms are closer than $3 \AA$ and are labelled as solid-like whenever their $\bar{q}_{3}$ is larger than 0.28 . 


\section{Cluster size and potential energy versus time for all system sizes and model potentials studied}

In order to determine the temperature at which the cluster was critical, we evaluated the highest temperature at which the cluster grows and the lowest temperature at which it melts. In Fig. 4 of the main text we represented the number of molecules in the ice cluster versus time for system H simulated with TIP4P/2005 potential. In what follows, we present the results of the cluster size versus time for all sizes (B,L and H) for both the TIP4P/2005 (Fig 10) and TIP4P/Ice water models (Fig 11). An analogous result could have been obtained by monitoring the potential energy of the system as a function of time (see the right panels at Figs. 10 and 11).
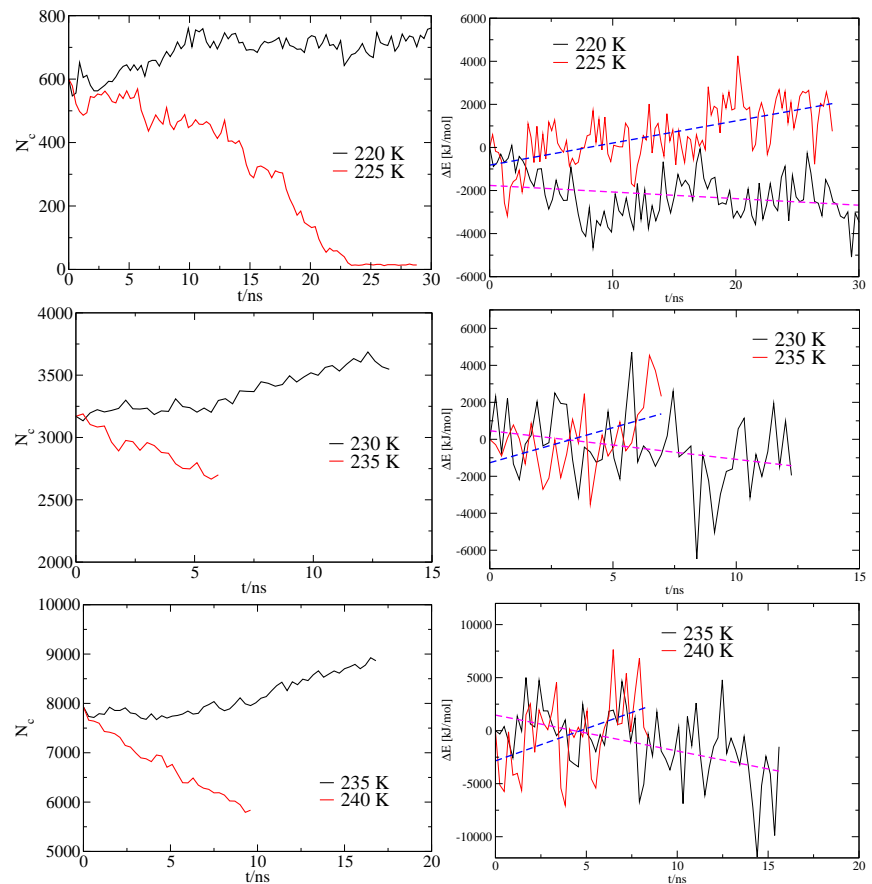

FIG. 10: Left-hand panels: Number of molecules in the ice cluster versus time for system $\mathrm{B}$ (top), L (middle) and $\mathrm{H}$ (bottom) simulated with the TIP4P/2005 potential. Righthand panels: energy difference (between the energy at time $t$ and the one at time zero) versus time. Results are shown for different temperatures as indicated in the legend.

The energy is much less sensitive to changes in the cluster size than the order parameter. This is due to the fact that the number of molecules in the cluster is a small fraction of the total number of molecules. Nonetheless, by making a linear fit to the time evolution of the energy we obtain in all cases consistent results with the analysis based in the order parameter: the cluster grows when the slope is negative and shrinks when it is positive.
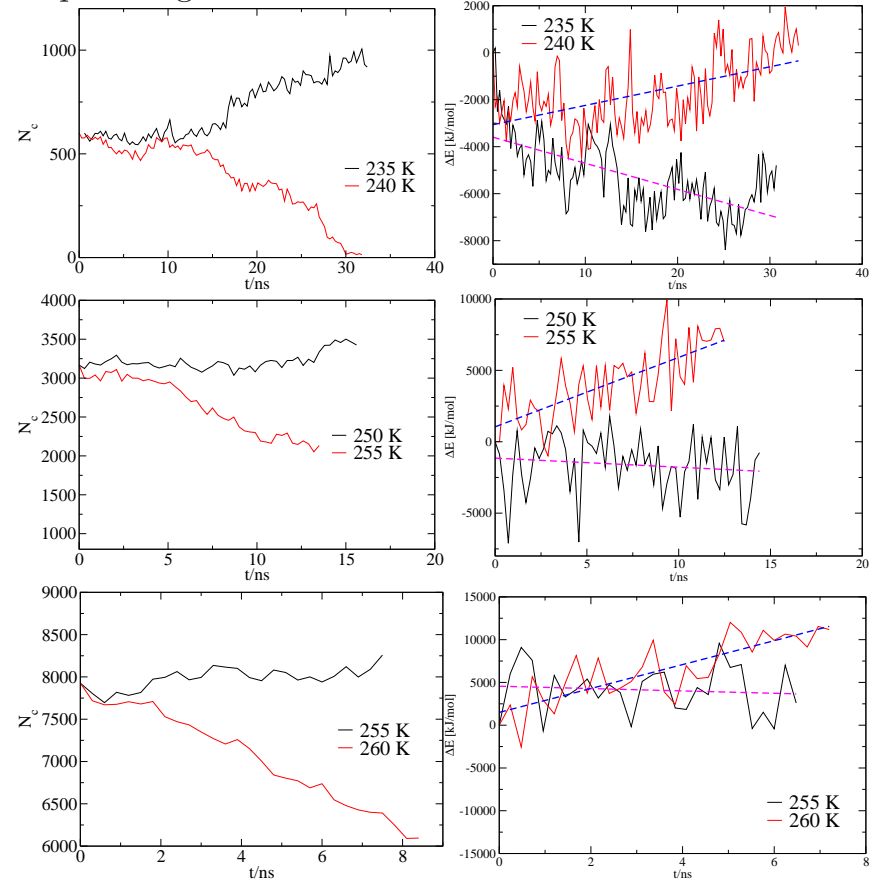

FIG. 11: Same as Fig. 10 but for TIP4P/Ice instead of TIP4P/2005.

\section{ACKNOWLEDGMENTS}

E. Sanz acknowledges financial support from the EU grant 322326-COSAAC-FP7-PEOPLE-2012-CIG and from the Spanish grant Ramon y Cajal. C. Valeriani acknowledges financial support from the EU grant 303941ANISOKINEQ-FP7-PEOPLE-2011-CIG and from the Spanish grant Juan de la Cierva. Fundings also come from MECD Project FIS2010-16159 and from CAM MODELICO P2009/ESP/1691. All authors acknowledge the use of the super-computational facility Tirant at Valencia from the Spanish Supercomputing Network (RES), along with the technical support and the generous allocation of CPU time to carry out this project (through projects QCM-2012-2-0017, QCM-2012-3-0038 and QCM-2013-1-0047). One of us (C. Vega) would like to dedicate this paper to the memory of Prof. Tomas Boublik. We thank the three referees for their useful comments and to A. Reinhardt and J.P.K. Doye for sending us a preprint of their work prior to publication.
[1] P. G. Debenedetti, Metastable liquids: Concepts and Principles (Princeton University Press, 1996).

[2] D. Kashchiev, Nucleation: Basic Theory with Applications (Butterworth-Heinemann, Oxford, 2000).
[3] W. Cantrell and A. Heymsfield, Bull. Amer. Meteor. Soc. 86, 795 (2005).

[4] M. B. Baker, Science 276, 1072 (1997).

[5] P. J. DeMott, A. J. Prenni, X. Liu, S. M. Kreidenweis, 
M. D. Petters, C. H. Twohy, M. S. Richardson, T. Eidhammer, and D. C. Rogers, Proc. Nat. Acad. Sci. USA 107, 11217 (2010).

[6] G. J. Morris and E. Acton, Cryobiology 66, 85 (2013).

[7] S. S. Hirano and C. D. Upper, Microbiol. Molec. Biol. Rev. 64, 624 (2000).

[8] L. R. Maki, E. L. Galyan, C. M.M., and D. R. Caldwell, Appl. Microbiol. 28, 456 (1974).

[9] J. K. Li and T. C. Lee, Trends in Food Sci. \& Tech. 6, 259 (1995).

[10] A. Michaelides and K. Morgenstern, Nature Materials 6, 597 (2007).

[11] A. G. Gerrard, Rocks and Landforms (Springer Netherlands, 1988).

[12] H. R. Pruppacher, J. Atmosph. Sci. 52, 1924 (1995).

[13] P. Taborek, Phys. Rev. B 32, 5902 (1985).

[14] D. A. Knopf and Y. J. Rigg, J. Phys. Chem. A 115, 762 (2011).

[15] E. B. Moore and V. Molinero, Nature 479, 506 (2011).

[16] T. Li, D. Donadio, G. Russo, and G. Galli, Phys. Chem. Chem. Phys. 13, 19807 (2011).

[17] B. Riechers, F. Wittbracht, A. Hutten, and T. Koop, Phys. Chem. Chem. Phys. 15, 5873 (2013).

[18] B. Kramer, O. Hubner, H. Vortisch, L. Woste, T. Leisner, M. Schwell, E. Ruhl, and H. Baumgartel, J. Chem. Phys. 111, 6521 (1999).

[19] P. Stockel, I. M. Weldinger, H. Baumgartel, and T. Leisner, J. Phys. Chem. A 109, 2540 (2005).

[20] B. J. Murray, S. L. Broadley, T. W. Wilson, S. J. Bull, R. H. Wills, H. K. Christenson, and E. J. Murray, Phys. Chem. Chem. Phys. 12, 10380 (2010).

[21] O. Mishima, J. Chem. Phys. 133, 144503 (2010).

[22] P. G. Debenedetti, J. Phys. Condens. Matter 15, R1669 (2003).

[23] A. Mori, M. Maruyama, and Y. Furukawa, J. Phys. Soc. Japan 65, 2742 (1996).

[24] C. A. Jeffery and P. H. Austin, J. Geophys. Research 102, 25269 (1997).

[25] S. B. Kiselev, Int. J. Thermophys. 22, 1421 (2001).

[26] J. Liu, C. E. Nicholson, and S. J. Cooper, Langmuir 23, 7286 (2007).

[27] P. R. ten Wolde and D. Frenkel., Science 277, 1975 (1997).

[28] S. Auer and D. Frenkel, Nature 409, 1020 (2001).

[29] R. P. Sear, Internat. Materials Rev. 57, 328 (2012).

[30] I. Svishchev and P. G. Kusalik, Phys. Rev. Lett. 73, 975 (1994).

[31] K. Koga, G. T. Gao, H. Tanaka, and X. C. Zeng, Nature 412, 802 (2001).

[32] E. B. Moore, E. de la Llave, K. Welke, D. A. Scherlis, and V. Molinero, Phys. Chem. Chem. Phys. 12, 4124 (2010).

[33] L. Vrbka and P. Jungwirth, J. Phys. Chem. B 110, 18126 (2006).

[34] E. B. Moore and V. Molinero, J. Chem. Phys. 132, 244504 (2010).

[35] E. B. Moore and V. Molinero, Phys. Chem. Chem. Phys. 13, 20008 (2011).

[36] M. Matsumoto, S. Saito, and I. Ohmine, Nature 416, 409 (2002).

[37] M. Yamada, S. Mossa, H. E. Stanley, and F. Sciortino, Phys. Rev. Lett. 88, 195701 (2002).

[38] T. A. Kesselring, E. Lascaris, G. Franzese, S. V. Buldyrev, H. J. Hermann, and H. E. Stanley, J. Chem.
Phys. 138, 244506 (2013).

[39] R. Radhakrishnan and B. Trout, J. Am. Chem. Soc 125, 7743 (2003).

[40] R. Radhakrishnan and B. L. Trout, Phys. Rev. Lett. 90, 158301 (2003).

[41] D. Quigley and P. M. Rodger, J. Chem. Phys. 128, 154518 (2008).

[42] A. V. Brukhno, J. Anwar, R. Davidchack, and R. Handel, J. Phys. Condens. Matter 20, 494243 (2008).

[43] A. Reinhardt and J. P. K. Doye, J. Chem. Phys. 136, 054501 (2012).

[44] A. Reinhardt and J. P. K. Doye, The Journal of Chemical Physics 139, 096102 (2013).

[45] B. Zobrist, T. Koop, B. P. Luo, C. Marcolli, and T. Peter, J. Phys. Chem. C 111, 2149 (2007).

[46] P. A. Alpert, J. Y. Aller, and D. A. Knopf, Phys. Chem. Chem. Phys. 13, 19882 (2011).

[47] S. C. Hardy, Philos. Mag. 35, 471 (1977).

[48] R. L. Davidchack, R. Handel, J. Anwar, and A. V. Brukhno, J. Chem. Theory Comput. 8, 2383 (2012).

[49] J. L. F. Abascal and C. Vega, J. Chem. Phys. 123, 234505 (2005).

[50] J. L. F. Abascal, E. Sanz, R. G. Fernandez, and C. Vega, J. Chem. Phys. 122, 234511 (2005).

[51] C. Vega and J. L. F. Abascal, Phys. Chem. Chem. Phys 13, 19663 (2011).

[52] X. M. Bai and M. Li, J. Chem. Phys. 124, 124707 (2006).

[53] A. J. C. Ladd and L. Woodcock, Molec. Phys. 36, 611 (1978).

[54] R. G. Pereyra, I. Szleifer, and M. A. Carignano, J. Chem. Phys. 135, 034508 (2011).

[55] L. C. Jacobson and V. Molinero, J. Am. Chem. Soc 133, 6458 (2011).

[56] B. C. Knott, V. Molinero, M. F. Doherty, and B. Peters, J. Am. Chem. Soc. 134, 19544 (2012).

[57] S. Auer and D. Frenkel, Ann. Rev. Phys. Chem. 55, 333 (2004).

[58] S. Auer and D. Frenkel, Nature 409, 1020 (2001).

[59] R. J. Allen, P. B. Warren, and P. R. ten Wolde, Phys. Rev. Lett. 94, 018104 (2005).

[60] P. G. Bolhuis, D. Chandler, C. Dellago, and P. L. Geissler, Ann. Rev. Phys. Chem. 53, 291 (2002).

[61] M. Volmer and A. Weber, Z. Phys. Chem. 119, 277 (1926).

[62] R. Becker and W. Doring, Ann. Phys. 24, 719 (1935).

[63] K. F. Kelton, Crystal Nucleation in Liquids and Glasses, vol. 45 (Academic Press, Boston, 1991).

[64] S. Auer and D. Frenkel, J. Chem. Phys. 120, 3015 (2004).

[65] B. Hess, C. Kutzner, D. van der Spoel, and E. Lindahl, J. Chem. Theory Comput. 4, 435 (2008).

[66] C. Vega, J. L. F. Abascal, M. M. Conde, and J. L. Aragones, Faraday Discuss. 141, 251 (2009).

[67] H. L. Pi, J. L. Aragones, C. Vega, E. G. Noya, J. L. F. Abascal, M. A. Gonzalez, and C. McBride, Molec. Phys. 107, 365 (2009).

[68] M. A. Gonzalez and J. L. F. Abascal, J. Chem. Phys. 132, 096101 (2010).

[69] U. Essmann, L. Perera, M. L. Berkowitz, T. Darden, H. Lee, and L. G. Pedersen, J. Chem. Phys. 103, 8577 (1995).

[70] M. Parrinello and A. Rahman, J. Appl. Phys. 52, 7182 (1981). 
[71] G. Bussi, D. Donadio, and M. Parrinello, J. Chem. Phys. 126, 014101 (2007).

[72] W. Lechner and C. Dellago, J. Chem. Phys. 129, 114707 (2008).

[73] A. Reinhardt, J. P. K. Doye, E. G. Noya, and C. Vega, J. Chem. Phys. 137, 194504 (2012).

[74] L. M. Ghiringhelli, C. Valeriani, J. H. Los, E. J. Meijer, A. Fasolino, and D. Frenkel, Molec. Phys. 106, 2011 (2008).

[75] T. Li, D. Donadio, L. M. Ghiringhelli, and G. Galli, Nature Mat. 8, 726 (2009).

[76] A. Bogdan, The Journal of Chemical Physics 106, 1921 (1997).

[77] C. Vega and E. de Miguel, J. Chem. Phys. 126, 154707 (2007).

[78] E. Sanz, C. Vega, J. L. F. Abascal, and L. G. MacDowell, Phys. Rev. Lett. 92, 255701 (2004).

[79] T. Koop, B. Luo, A. Tslas, and T. Peter, Nature 406, 611 (2000).

[80] E. G. Noya, C. Menduina, J. L. Aragones, and C. Vega, J. Phys. Chem. C 111, 15877 (2007).

[81] P. Kumar, S. V. Buldyrev, S. R. Becker, P. H. Poole, F. W. Starr, and H. E. Stanley, Proc. Nat. Acad. Sci. 104, 9575 (2007).

[82] D. Frenkel and B. Smit, Understanding Molecular Simulation (Academic Press, London, 1996).

[83] L. Granasy, T. Pusztai, and P. F. James, J. Chem. Phys. 117, 6157 (2002).

[84] R. Handel, R. L. Davidchack, J. Anwar, and A. Brukhno, Phys. Rev. Lett. 100, 036104 (2008).

[85] H. W. Horn, W. C. Swope, J. W. Pitera, J. D. Madura, T. J. Dick, G. L. Hura, and T. Head-Gordon, J. Chem. Phys. 120, 9665 (2004).

[86] J. L. F. Abascal and C. Vega, J. Chem. Phys. 133, 234502 (2010).

[87] J. L. F. Abascal and C. Vega, J. Chem. Phys. 134, 186101 (2011).

[88] K. T. Wikfeldt, C. Huang, A. Nilsson, and L. G. M.
Pettersson, J. Chem. Phys. 134, 214506 (2011).

[89] L. Filion, M. Hermes, R. Ni, and M. Dijkstra, J. Chem. Phys. 133, 244115 (2010).

[90] S. E. M. Lundrigan and I. Saika-Voivod, J. Chem. Phys. 131, 104503 (2009).

[91] R. Shevchuk and F. Rao, J. Chem. Phys. 137, 036101 (2012).

[92] S. D. Overduin and G. N. Patey, J. Chem. Phys. 138, 184502 (2013).

[93] D. T. Limmer and D. Chandler, J. Chem. Phys. 138, 214504 (2013).

[94] P. H. Poole, R. K. Bowles, I. Saika-Voivod, and F. Sciortino, J. Chem. Phys. 138, 034505 (2013).

[95] Y. Liu, J. C. Palmer, A. Z. Panagiotopoulos, and P. G. Debenedetti, J. Chem. Phys. 137, 214505 (2012).

[96] R. Pruppacher and J. D. Klett, Microphysics of Clouds and Precipitation (Kluwer Academic Publishers, 1997).

[97] R. J. Speedy and C. A. Angell, J. Chem. Phys. 65, 851 (1976).

[98] D. E. Hare and C. M. Sorensen, J. Chem. Phys. 87, 4840 (1987).

[99] V. Holten, C. E. Bertrand, M. A. Anisimov, and J. V. Sengers, J. Chem. Phys. 136, 094507 (2012).

[100] V. Molinero and E. B. Moore, J. Phys. Chem. B 113, 4008 (2009).

[101] C. Vega, M. Martin-Conde, and A. Patrykiejew, Mol. Phys. 104, 3583 (2006).

[102] M. M. Conde, C. Vega, and A. Patrykiejew, J. Chem. Phys. 129, 014702 (2008).

[103] L. S. Bartell and J. F. Huang, J. Phys. Chem. 98, 7455 (1994).

[104] J. F. Huang and L. S. Bartell, J. Phys. Chem. 99, 3924 (1995).

[105] M. W. Mahoney and W. L. Jorgensen, J. Chem. Phys. 112, 8910 (2000).

[106] M. S. G. Razul and P. G. Kusalik, J. Chem. Phys. 134, 014710 (2011). 